\title{
Características de comportamiento sexual en hombres de la Ciudad de México
}

\author{
Carlos Hernández-Girón, M.C., M. en C., ${ }^{(1)}$ Aurelio Cruz-Valdez, M.C., M. en C., ${ }^{(1)}$ Manuel Q uiterio-Trenado, Biól., ${ }^{(1)}$
} Armando Peruga, M.D., Ph.D., ${ }^{(2)}$ Mauricio Hernández-Avila, M.D., D r.Sc.(1)

\section{Hernández-Girón C, Cruz-Valdez A, Quiterio-Trenado M, Peruga A, Hernández-Avila $M$. Características de comportamiento sexual en hombres de la Ciudad de México. Salud Publica Mex 1999;41:95-100.}

\section{Resumen}

Objetivo. Determinar las principales características de comportamiento sexual en hombres de la Ciudad de México, incluyendo número de parejas sexuales, antecedentes de enfermedades de transmisión sexual (ETS) y creencias acerca del síndrome de inmunodeficiencia adquirida (SIDA). Ma terial y métodos. Durante 1995 se realizó un estudio epidemiológico transversal, basado en un muestreo multietápico por conglomerados; se entrevistó a 1377 hombres de 15 a 49 años de edad, a quienes se les aplicó un cuestionario estructurado. Para el análisis estadístico de los datos se realizaron pruebas de significancia como t de Student y $\chi^{2}$. Resultados. Entre los entrevistados la media de edad fue de 34.5 años ( $D E=7.5$ años), y la media de inicio de relaciones sexuales de 17.7 años ( $D E=2.8$ años). La proporción global de uso de condón durante la última relación sexual fue de 24.6\%; de acuerdo con el tipo de pareja sexual femenina en el último año, 18.8\% lo usaron con parejas regulares y $62.5 \%$ con múltiples parejas. El $2 \%$ refirió síntomas sugestivos de uretritis gonocócica. Finalmente $97.5 \%$ refirió saber qué significaba la palabra SIDA y conocer ciertas medidas protectoras contra la infección. Conclusiones En este estudio se identificaron características de comportamiento sexual riesgoso para adquirir y transmitir alguna ETS, como tener múltiples parejas sexuales, no usar el condón y presentar antecedentes de ETS.

Palabras clave: conducta sexual; condones; hombres; México
Hernández-Girón C, Cruz-Valdez A, Quiterio-Trenado M, Peruga A,

Hernández-Avila $M$.

Characteristics of sexual behavior

in the male population from Mexico City.

Salud Publica Mex 1999;41:95-100.

\begin{abstract}
A bstract
Objective.To determine the major features of sexual behavior in men from Mexico City, such as the number of sexual partners, history of sexually transmitted disease (STD) and beliefs on AIDS. Material and methods A transversal epidemiologic study was conducted in 1995 based on multistage sampling with conglomerates. A total of 1377 males from 15 to 49 years of age was interviewed by means of a structured questionnaire. Statistics such as Student's test and $\chi^{2}$ were applied to determine significance. Results. Subject mean age was 17.7 years $(S D=2.8$ years) 0 verall proportion of condom use in the last intercourse was $24.6 \%$ depending on the type of partner : $18.8 \%$ used it with regular partners and $62.5 \%$ with multiple partners. Symptoms suggesting gonococcal uretritis were found in $2 \%$. Finally, $97.5 \%$ understood the meaning of AIDS and knew some protective measures against infection. Conclusions So me risky characteristics of sexual behavior were identified concerning the transmission of STD such as multiple sexual partners, not using condom and STD antecedents.
\end{abstract}

Key words: sex behavior; condoms; men; Mexico

(1) Centro de Investigación en Salud Poblacional, Instituto N acional de Salud Pública, México.

(2) O ficina Sanitaria Panamericana, O rganización Panamericana de la Salud.

Fecha de recibido: 16 de julio de 1998 • Fecha de aprobado: 19 de octubre de 1998

Solicitud de sobretiros: Dr. Aurelio Cruz Valdez. Centro de Investigación en Salud Poblacional. Instituto N acional de Salud Pública. Av. Universidad 655, colonia Sta. María A huacatitlán, 62508 Cuernavaca, Morelos, México. Correo electrónico: acruz@ insp3.insp.mx 
S e estima que en el mundo se producen anualmen$S$ te 250 millones de nuevos casos de alguna enfermedad de transmisión sexual (ETS). Se calcula que para el año 2000 habrá cerca de 38 millones de infectados por el virus de la inmunodeficiencia humana (VIH), de los cuales una cuarta parte corresponderá a América Latina. En México, la cifra de casos notificados (acumulados) de SIDA hasta el 1 de enero de 1998 era de 33 632; sin embargo, al corregirla de acuerdo con el subregistro y el retardo en la notificación de los casos, se estima que aumenta a aproximadamente 53000 . Del total de casos, $56 \%$ se presentó en el Distrito Federal (D.F.), el Estado de México y Jalisco. ${ }^{1}$

Hasta la fecha no existen vacunas ni tratamientos eficaces contra ciertas ETS -principalmente contra las de origen viral-, de tal manera que la prevención basada en la educación sexual y en la utilización de métodos anticonceptivos de barrera es la principal estrategia para controlar las epidemias de SIDAy de otras ETS. Para lograr lo anterior es necesario conocer las principales pautas del comportamiento sexual de la población general, blanco sobre el cual se pretende impactar con las estrategias de control y prevención de ETS.

En México organizaciones como el Consejo Nacional de Prevención y Control del SIDA (CONASIDA) y la Fundación Mexicana para la Salud (FUNSALUD), han realizado diversas investigaciones como, por ejemplo, la Encuesta sobre Comportamiento Sexual en la Ciudad de México, realizada entre 1992 y 1993 por CONASIDA. ${ }^{2}$ Los resultados sobre comportamiento sexual en hombres de la Ciudad de México que ofrece esa encuesta se han comparado con los que se obtuvieron en esta investigación.

En este estudio se informa acerca de las características del comportamiento sexual de los hombres de la Ciudad de México, incluyendo el tipo y el número de parejas sexuales, así como los antecedentes de ETS y las creencias sobre el SIDA, elemento que permitirá establecer nuevas estrategias de control y prevención de ETS en la población general, en especial entre los jóvenes.

\section{Material y métodos}

Durante 1995 se realizó un estudio transversal con base poblacional en hombres de 15 a 49 años residentes en la Ciudad de México. Para seleccionar la población en estudio, se utilizó el Marco Muestral Maestro del Sistema Nacional de Encuestas de Salud de la Dirección General de Epidemiología, y se realizó un muestreo probabilístico multietápico de la siguiente manera: como unidades primarias de muestreo (UPM), se conside- raron a las 16 delegaciones del D.F. Las unidades de primera etapa (UPE) fueron las áreas geoestadísticas básicas (AGEB) seleccionadas por cada delegación. Posteriormente se obtuvieron las unidades de segunda etapa (USE), tomando en cuenta las manzanas en forma proporcional a las AGEB correspondientes. Como unidades de tercera etapa (UTE) se consideró a los conglomerados de viviendas, incluyendo todos los hogares que los conformaban. Por último, como unidad de análisis se consideró a un hombre entre 15 y 49 años de edad, por cada vivienda seleccionada.

Las delegaciones del D.F. (UPM) se sometieron a estratificación según sus características sociodemográficas, a fin de incrementar la eficiencia del diseño. Se obtuvo una muestra de 3091 viviendas donde era probable encontrar un hombre en el rango de edad esperado. En aquellas viviendas con más de un hombre de entre 15 y 49 años, se utilizó una hoja de números aleatorios para seleccionar sólo a uno.

Para la recolección de información se elaboró un cuestionario que comprendía dos secciones:

Hogar: conformado por 16 preguntas para identificar características sociodemográficas de la vivienda y de la familia.

Individual: conformado por 64 preguntas para conocer las características de comportamiento sexual, antecedentes y conocimientos sobre ETS/SIDA.

El cuestionario se validó con dos pruebas piloto utilizando AGEB no contempladas en el marco muestral: una realizada en la ciudad de Cuernavaca y otra en la Ciudad de México. Previa elaboración de manuales de codificación y captura, la información recolectada se procesó en la Dirección de Informática de la Secretaría de Salud, utilizando el paquete Foxpro2. Como criterios para la validez y la calidad de la información, además del sistema de doble captura de los datos, se usó uno de "llaves de identificación" que comprendía la hoja frontal de cada cuestionario.

Se creó una variable de comportamiento sexual denominada tipo de pareja sexual, mediante la cual se identificó a los hombres según el tipo de pareja sexual femenina que habían tenido durante el último año:

1. Estables: relaciones con una sola pareja formal (esposa o novia).

2. Parejas regulares: relaciones durante un año o más con alguna pareja regular, además de su pareja formal (esposa o novia).

3. Parejas múltiples: relaciones con diversas parejas durante menos de un año. 
El índice de nivel socioeconómico (NSE) se elaboró según la propuesta de M. Bronfman y colaboradores. $^{3}$

En el plan de análisis estadístico se utilizaron los paquetes Stata 3.1, SPSS/pc 4.0, y EGRET, para realizar pruebas estadísticas de significancia ( $t$ de Student y $\left.\chi^{2}\right){ }^{4-6}$

\section{Resultados}

En la muestra de 3091 viviendas seleccionadas se encontraron 2739 hombres de entre 15 y 49 años de edad (tasa de efectividad $=88.6 \%$ ), residentes en la Ciudad de México, de los cuales 50.3\% (1377/2 739) contestaron ambas secciones del cuestionario; $32.8 \%$ (897/2 739) se clasificaron como ausentes temporales, esto es, hombres seleccionados a quienes fue imposible localizar aun después de cinco visitas domiciliarias, y $16.9 \%$ $(465 / 2739)$ se negaron a contestar.

La media de edad en la población estudiada fue de 34.5 años ( $\mathrm{DE}=7.5$ años); $81.6 \%$ de estos individuos estaban casados (1 124/1 377); 80.7\% habían terminado sus estudios de secundaria; y los años de escolaridad referidos por los entrevistados comprendían desde cero hasta 25 años (media de 10.7 años, $\mathrm{DE}=4.6$ años). De los entrevistados $54.7 \%$ correspondieron a un NSE medio; 33\%, a NSE alto, y $12.3 \%$, a NSE bajo. Al estratificar por tipo de parejas sexuales no hubo diferencias significativas.

Del total de entrevistados, $99.4 \%$ (1369/1377) manifestaron que habían tenido relaciones sexuales alguna vez en su vida. La media de edad de inicio de relaciones sexuales fue de 17.7 años ( $\mathrm{DE}=2.8$ años).

Por otra parte, se identificaron tres grupos de acuerdo con el tipo de parejas sexuales que habían tenido en el último año: monógamos, 79.4\% (1047/1319); con múltiples parejas, $15 \%$ (192/1 319), y con alguna pareja regular, 6.1\% (80/1 319) (figura 1, cuadro I).

La frecuencia general de utilización del condón en la última relación sexual fue de $26.4 \%$ (343/1300). Según el tipo de pareja, la frecuencia fue de $62.5 \%$ (120/192) con múltiples parejas, 36.2\% (29/80) con parejas regulares y 18.9\% (194/1 029) monógamos (figura 2).

Los principales motivos por los cuales los individuos con parejas regulares utilizaron el condón en la última relación sexual fueron la planificación familiar $(86.7 \%)$ y la prevención de ETS (12.1\%), mientras que para aquellos con múltiples parejas fueron la prevención de ETS $(87.5 \%)$ y la planificación familiar $(9.2 \%)$ (figura 3).

Durante la última relación con una pareja ocasional, 83 entrevistados usaron al menos un condón; de

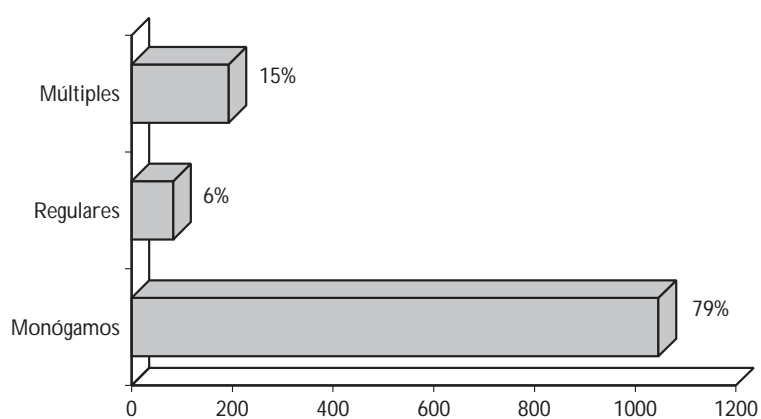

Figura 1.Tipo de pareja sexual femenina. Hombres de la Ciudad de México, 1995

\section{Cuadro I \\ Características de comportamiento sexual SEGÚN TIPO DE PAREJA SEXUAL EN 1377 HOMBRES. Ciudad de México, 1995}

Variable

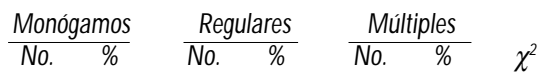

Edad a la primera relación sexual (años)

\begin{tabular}{lrrrrrrr}
\hline $10-14$ & 75 & 7.2 & 15 & 18.8 & 25 & 13.0 & 0.093 \\
\hline $15-19$ & 717 & 68.5 & 51 & 63.8 & 125 & 65.1 & \\
\hline $20-24$ & 219 & 20.9 & 13 & 16.2 & 37 & 19.3 & \\
\hline 25 -más & 36 & 3.4 & 1 & 1.2 & 5 & 2.6 & \\
\hline Total & 1047 & 100.0 & 80 & 100.0 & 192 & 100.0 &
\end{tabular}

Ultima relación sexual con pareja regular

\begin{tabular}{lrrrrrrr} 
Ultima semana & 762 & 74.1 & 50 & 63.3 & 111 & 57.8 & 0.000 \\
\hline Ultimo mes & 226 & 22.0 & 26 & 32.9 & 34 & 17.7 & \\
\hline Ultimo año & 40 & 3.9 & 3 & 3.8 & 47 & 24.5 & \\
\hline Total & 1028 & 100.0 & 79 & 100.0 & 192 & 100.0 &
\end{tabular}

Utilizó un condón en el último contacto sexual

\begin{tabular}{lrrrrrrr} 
N o & 835 & 81.1 & 50 & 63.3 & 72 & 37.5 & 0.000 \\
\hline Sí & 194 & 18.9 & 29 & 36.7 & 120 & 62.5 & \\
\hline Total & 1029 & 100.0 & 79 & 100.0 & 192 & 100.0 &
\end{tabular}

N ota: los totales varían de acuerdo con los valores perdidos

ellos, $72 \%$ lo obtuvieron en farmacias, y el resto, en bares y hoteles.

También se indagó sobre antecedentes de sintomatología de ETS en el último año: 2\% (28/1377) de los entrevistados refirieron síntomas de disuria y/o piuria, y $0.5 \%$ (7/1377), algún signo de ulceración genital. Mientras duraron dichos síntomas y signos, $62 \%$ $(18 / 29)$ de los individuos modificaron sus prácticas sexuales, entre las cuales predominó la abstinencia sexual en $77.8 \%$ de los sujetos (14/18). 


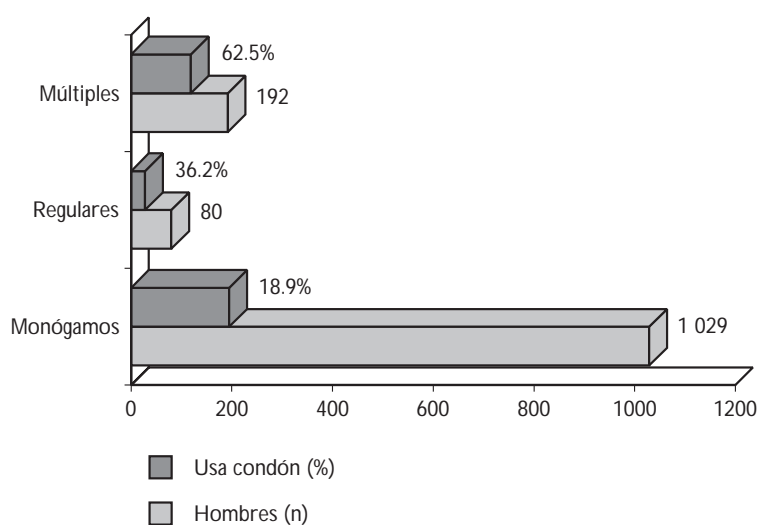

Figura 2. UTILIZACIÓN DE CONDÓN SEGÚN TIPO de PAREja sexual femenina. Hombres de la Ciudad de México, 1995

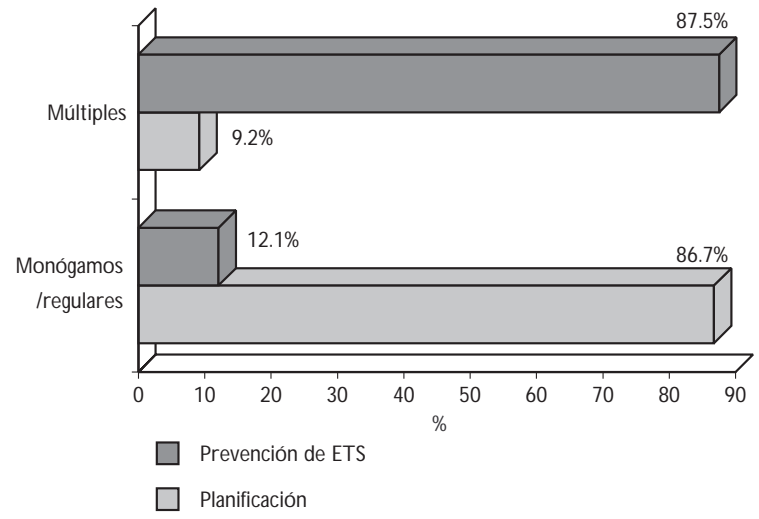

Figura 3. Motivo principal para usar CONdón SEgÚN tipo de pareja sexual femenina. Hombres de la Ciudad DE MéxıICo, 1995

Por otra parte, $97.5 \%$ (1343/1377) de los entrevistados indicaron que sabían lo que significaba el término SIDA, y $11.8 \%$ (163/1377) conocían a alguien que lo padecía; $47 \%$ habían utilizado un condón alguna vez en su vida. La mayoría conocía dónde adquirirlos: $92.4 \%$ hicieron referencia a las farmacias; $26.7 \%$, a los centros de salud, y $17.3 \%$, a los hospitales y clínicas.

Finalmente se hicieron preguntas para evaluar los conocimientos acerca de las medidas preventivas. De éstas se reconocieron como verdaderas para prevenir el SIDA: la utilización de agujas estériles en las inyecciones $(97.5 \%)$, el uso del condón durante las relaciones sexuales $(85.2 \%)$ y la fidelidad con la pareja $(83.9 \%)$. Las principales medidas reconocidas como falsas en la prevención del SIDA fueron una buena dieta alimenticia (90.5\%), evitar tocar a alguien infectado (87.2\%), evitar compartir alimentos con alguien infectado (85.8\%) y evitar baños públicos $(71.7 \%)$. Estas respuestas se estratificaron por tipo de pareja, y no se encontraron diferencias estadísticamente significativas.

\section{Discusión}

En este estudio predominó el grupo de 30 a 39 años de edad (44\%), a diferencia de lo que se observa en el Censo General de Población para la Ciudad de México,? donde abundan los grupos de 15 a $19(21.5 \%$ ) y 20 a 24 años (19.6\%); aparece una diferencia importante que puede atribuirse al diseño muestral $y$, consecuentemente, al efecto de la tasa de no respuesta.

La mayoría de los entrevistados eran casados; sólo $4.8 \%$ eran solteros, mientras que los datos del Censo de 1990 reflejan una paridad entre casados $(43.7 \%$ ) y solteros $(46.6 \%)$. Esta diferencia se puede atribuir a que la mayor proporción de no respuesta $(65.2 \%)$ y ausentes temporales $(68 \%)$ correspondió a los solteros.

Los hallazgos de esta investigación son similares a los de la Encuesta sobre Comportamiento Sexual 1992-93 que llevó a cabo CONASIDA en la Ciudad de México; en la misma se indica que las generaciones más recientes recurren a métodos de protección cuando tienen relaciones sexuales, para prevenir tanto un embarazo como alguna ETS. ${ }^{2}$ En un estudio realizado con estudiantes de secundaria en Honduras, se encontró que los sujetos pertenecientes a los niveles socioeconómicos medio y alto ${ }^{8}$ poseían un mayor conocimiento y hacían más uso de medidas preventivas (incluyendo el condón) contra ETS/SIDA.

Se encontró que la edad media de inicio de relaciones sexuales era de 17.7 años; al respecto, otros estudios muestran resultados diversos, lo que se debe principalmente a las distintas metodologías empleadas. Por ejemplo, una encuesta reproductiva que se aplicó en población adolescente urbana y rural de varios países de América Latina entre 1984 y 1987, demostró que la edad media de inicio de relaciones sexuales en mujeres variaba de 16.6 años en Brasil y Costa Rica, hasta 17 años en México y El Salvador. La media de inicio de relaciones sexuales en hombres iba de 14.3 años en Jamaica hasta 15.7 años en México. ${ }^{9}$ Por otra parte, la encuesta sobre comportamiento sexual mencionada con anterioridad notificó una edad de inicio de relaciones sexuales de 17.4 años. $^{2}$

La proporción general de utilización del condón fue de $24.6 \%$; esta proporción fue mayor entre los hombres con parejas múltiples $(62.5 \%)$ que en aquellos con 
parejas regulares $(36.7 \%)$. Diversos estudios internacionales indican resultados similares al respecto; así por ejemplo, Rhodes y colaboradores llevaron a cabo un estudio transversal en dos ciudades, en Gran Bretaña, para determinar la prevalencia de infección por VIH y comportamiento sexual en personas drogadictas; la frecuencia de uso del condón en hombres con parejas múltiples y regulares en Londres fue de 66 y $48 \%$, respectivamente, en comparación con 30 y $25 \%$, respectivamente, de Glasgow. ${ }^{10}$ En Estados Unidos de América (EUA), en una muestra de hombres heterosexuales de origen hispano, Sabogal y colaboradores ${ }^{11}$ notificaron una prevalencia de uso del condón con parejas múltiples y regulares de 30 y $20 \%$, respectivamente. Por otra parte, y también en EUA, en una muestra de hombres que acudieron a un centro de tratamiento de ETS, $43 \%$ de ellos utilizaron condón con parejas múltiples y $35 \%$ lo hicieron con parejas regulares. ${ }^{12}$ En México la frecuencia del uso del condón masculino muestra algunas similitudes; así, Izazola y colaboradores, en 1990 encontraron diferencias entre el uso del condón con múltiples parejas y con la pareja formal: 14 y $5 \%$, respectivamente, en heterosexuales 37 y $29 \%$ en bisexuales, y 25 y $9 \%$ en homosexuales. ${ }^{13}$

Las prevalencias de uretritis (síntomas de disuria y/o piuria, $2 \%$; ulceraciones genitales, $0.5 \%$ ) autoinformadas en el último año son inferiores a los resultados que ofrecen algunos estudios internacionales; así, en EUA, durante 1990, Shafer y colaboradores notificaron una prevalencia de alguna ETS actual y de antecedente de ETS en el último año transcurrido, entre adolescentes de 12 a 18 años recluidos en centros de detención, de 15 y $34 \%$, respectivamente. ${ }^{14}$ Un estudio realizado por Bochow y colaboradores ${ }^{15}$ durante 1991 en hombres homo y bisexuales de 10 países europeos, notificó una incidencia de cualquier ETS que no fuera por VIH, en los últimos 12 meses, de entre 2 y $3 \%$. Por otra parte, la encuesta sobre comportamiento sexual ya citada indicó una prevalencia de uretritis de 9.8\% (antecedente de disuria, en toda su vida). ${ }^{2}$ Estas diferencias se explican, primero, por el hecho de que los otros informes se refieren a grupos identificados con prácticas sexuales de riesgo $\mathrm{y}$, segundo, porque la temporalidad de antecedente de ETS indagada en otras encuestas era distinta.

El cuestionario se validó con dos pruebas piloto realizadas en las ciudades de Cuernavaca y México, lo que permitió hacer ajustes en el contenido y la estructura de las preguntas del cuestionario individual (principalmente sobre comportamiento sexual). A pesar de lo anterior, hubo la posibilidad de que ocurriera algún sesgo, por ejemplo de información (no diferencial) en la población encuestada, dadas las características de ciertas preguntas sobre su comportamiento sexual. En ocasiones la ausencia de privacidad pudo haber limitado y comprometido la veracidad de la información. Al calcular el tamaño de la muestra se hizo un sobremuestreo por edad y escolaridad, de tal manera que pudo haber mayor representación de los casados y mayores de 30 años, y menor representación de los solteros y menores de 20 años. Lo anterior pudo originar una subestimación de las variables estudiadas en estos grupos.

Si bien los resultados de esta investigación replican hallazgos de otros estudios nacionales e internacionales, es necesario manejarlos con prudencia al extrapolarlos a otras poblaciones basales. No obstante, se considera que estos resultados reflejan el comportamiento sexual de la población masculina de la Ciudad de México, principalmente de los hombres casados y que tienen entre 25 y 35 años de edad.

\section{Conclusiones}

A partir del surgimiento de los primeros casos de SIDA en México, se establecieron las medidas de control y prevención, enfocándolas inicialmente a grupos identificados como de alto riesgo para el contagio y la transmisibilidad de infección por VIH y otras ETS (homosexuales y trabajadoras sexuales). Con la creación de CONASIDA en 1986, estas medidas empezaron a difundirse a la población en general.

Sin embargo, la epidemia del SIDA va en aumento; cabe aquí tomar en cuenta varias consideraciones: el patrón de comportamiento de la infección por VIH se ha modificado, y hoy los casos en población heterosexual son más frecuentes. Debido a la historia natural del SIDA, los casos infectados hace 10 años comienzan a presentar signos de la enfermedad en la actualidad. Y, por último, la corrección en el subregistro y el retraso en la notificación de los casos ha incrementado las estadísticas.

El intento de modificar las prácticas sexuales de riesgo implica modificar el comportamiento sexual de los individuos, lo cual representa una tarea especialmente ardua. No obstante, el fortalecimiento de las campañas educativas de control y prevención de ETS es una tarea factible, cuyo impacto puede observarse ya en las actitudes y el conocimiento acerca de las ETS entre la población sexualmente activa, aparentemente sin riesgo de adquirir y transmitir alguna ETS.

Con los anteriores resultados se establece que existen características socioeconómicas (edad y nivel de escolaridad) y de comportamiento sexual (tipo de pa- 
rejas sexuales) similares a las encontradas en otros estudios nacionales e internacionales, que predicen la utilización del condón masculino.

Estos resultados sugieren que el uso del condón masculino depende de las características individuales (p.e., edad), y del conocimiento y las actitudes hacia las ETS (p.e., número de parejas sexuales) y del tipo de parejas sexuales (parejas múltiples, pareja regular), como lo indican otros estudios. ${ }^{15-17}$

Así entonces, es importante considerar que las campañas educativas, cuyo objetivo es fomentar la prevención de ETS, deben estar orientadas no sólo a grupos con prácticas de alto riesgo (trabajadoras sexuales, drogadictos intravenosos, homosexuales), sino también a sus clientes y población en general, particularmente a los jóvenes.

\section{Referencias}

1. Instituto $\mathrm{N}$ acional de Diagnóstico y Referencia Epidemiológicos. Boletín SIDA/ETS 1998;1(1):1-12.

2. Consejo $\mathrm{N}$ acional de Prevención y Control del SIDA.C omportamiento sexual en la Ciudad de México. Encuesta 1992-1993. México, D.F.:C O N ASIDA, 1994.

3. Bronfman $M, G$ uiscafré $H, C$ astro V, C astro R, Gutiérrez G. Medición de la desigualdad: una estrategia metodológica, análisis de las características socioeconómicas de la muestra. Arch Invest Med 1988;19:351-360. 4. Statistics and Epidemiology Research Corporation. EG RET: Epidemiological Graphics, Estimation and Testing Package. SERC, 909 N E. 3a. edición. Seattle: EG RET, 1990.

5. Stata Corporation. Stata Reference Manual: Release 3.1. 6a. edición. C 0llege Station TX: Stata Corporation, 1993.
6. N orusis $M . S P S S / p c=4.0$ base manual for the $\mathrm{IBMpc} / \mathrm{xt} /$ at $/$ and $\mathrm{ps} 2$. $\mathrm{C}$ hicago ILL: SPSS Inc, 1992.

7. Instituto $\mathrm{N}$ acional de Estadística, Geografía e Informática. Resultados definitivos.XI C enso G eneral de Población yVivienda. México, D.F.:IN EGI, 1990.

8. Machado-C astañeda H, López B, N olasco L, Zavala M, Cerrato Y. La actitud y el grado de conocimientos sobre el SIDA entre estudiantes de secundaria en Tegucigalpa, Honduras. En: A portfolio of AIDS/STD behavioral inter ventions and research. W ashington: O PS/O MS, 1992.

9. Morris L.Young adults in Latin A merica and the Caribbean:Their sexual experience and contraceptive use. Int Fam Plann Perspect 1988;14:153158.

10. Rhodes J, Bloor MJ, Donoghoe M, Haw S, Ettore B, Platt S et al. HIV prevalence and HIV risk behavior among injecting drug users in London and G lasgow. AIDS C are 1993;5(4):413-425

11. Sabogal F, Faigeles B, $C$ atania J. D ata from the $N$ ational A ID S behavioral survey II. Multiple sexual partners among Hispanics in high-risk cities. Fam Plann Perspect 1993;25(6):257-262.

12. Edwards S.A mong high-risk adults, men with more than four partners, women older than 19 use condoms less. Fam Plann Perspect 1992;24:8384.

13. Izazola JA, Gortmaker S, Basáñez R, Sepúlveda J. Relaciones sexuales entre mujeres y hombres identificados como homosexuales. Probabilidad de transmitir VIH a otras personas. En:VII Conferencia Internacional sobre SIDA; 1991; Florencia, Italia.

14. Shafer MA, Hilton J, Ekstrand M, Keogh J, Gee L, DiGiorgio L et al. Relationship between drug use and sexual behaviors and the ocurrence of STD's among high risk male youth. Sex Trans D is 1993;20(6):307-313. 15. Bochow M, Chiarotti F, Davies P, Dubois F, Dur W, Fouchard J et al. Sexual behavior of gay and bisexual men in ten European countries.AIDS Care 1994;6(5):533-549.

16. Pickering $H, Q$ uigley $M, H$ ayes $R$, Todd J, W ilkins A. D eterminants of condom use in 24000 prostitute/client contacts in the Gambia. AIDS 1993;7(8):1093-1098.

17. Morris M, Pramualratana A, Podhisita C, W aver M. The relational determinants of condom use with commercial sex partners in Thailand.AIDS 1995;9(5):507-515 